\title{
A geometric definition of the Mañé-Mather set and a Theorem of Marie-Claude Arnaud.
}

\author{
Patrick Bernard* and Joana Oliveira dos Santos ${ }^{\dagger}$
}

February 2011

Patrick Bernard, Université Paris-Dauphine, CEREMADE, UMR CNRS 7534

Pl. du Maréchal de Lattre de Tassigny

75775 Paris Cedex 16, France

patrick.bernard@ceremade.dauphine.fr
Joana O. Santos, Université Paris-Dauphine, CEREMADE, UMR CNRS 7534

Pl. du Maréchal de Lattre de Tassigny

75775 Paris Cedex 16, France

santos@ceremade. dauphine.fr

\begin{abstract}
We study some properties of Lipschitz exact Lagrangian manifolds isotopic to the zero section. We prove that if such a manifold is invariant under an optical Hamiltonian, then it must be a Lipschitz graph. This extends a recent result of Marie-Claude Arnaud. We also obtain a new geometric description of the Mañé-Mather invariant set.
\end{abstract}

Résumé. On étudie quelques propriétés des variétés exactes Lagrangiennes Lipschitz isotopes à la section nulle. On montre qu'une telle variété est un graphe Lipschitz si elle est invariante par un Hamiltonian optique, ce qui étend un résultat récent de Marie-Claude Arnaud. On obtient aussi une nouvelle description géométrique de l'ensemble invariant de Mañé-Mather.

Resumo Estudamos algumas propriedades das variedades Lagrangianas exactas Lipschitz isotópicas à secção nula. Mostramos que uma tal variedade, se for invariante por um Hamiltoniano óptico, é um gráfico Lipschitz, o que generaliza um resultado recente de Marie-Claude Arnaud. Obtemos também uma nova descrição geométrica do conjunto invariante de MañéMather.

MSC: 37J50, 37J05, 53D12, 57R17.

${ }^{*}$ membre de l'IUF

†author financed by the scholarship SFRH/BD/35773/2007 of the FCT, Portugal (Fundação para a Ciência e Tecnologia). 


\section{Introduction}

We study a $C^{2}$ Hamiltonian $H: T^{*} M \longrightarrow \mathbb{R}$ on the cotangent bundle of a compact manifold $M$ without boundary. Most of our results will be relevant only in the case where $H$ is optical, which means that it is convex with positive definite Hessian and superlinear in each fiber. Such Hamiltonians are also called Tonelli. We denote by $\mathcal{G}$ be the set of Lipschitz exact Lagrangian graphs in $T^{*} M$. An element of $\mathcal{G}$ is a subset $\Gamma \subset T^{*} M$ of the form

$$
\Gamma:=\{(x, d u(x)), x \in M\}
$$

where $u$ is a $C^{1,1}$ function (a $C^{1}$ function with Lipschitz differential). We will occasionally denote by $\mathcal{G}^{p}$ the set of $C^{p}$ exact Lagrangian Graphs. We denote by $\mathcal{H}^{p}$ the set of $C^{p}$ Hamiltonian diffeomorphisms of $T^{*} M$. An element of $\mathcal{H}^{p}$ is the time-one flow $\varphi_{G}^{1}$ of some $C^{p+1}$ time-dependent Hamiltonian function $G:[0,1] \times T^{*} M \longrightarrow \mathbb{R}$. It is well-known that $\mathcal{H}^{p}$ is a group of diffeomorphisms of $T^{*} M$. Finally, we introduce the set

$$
\mathcal{L}^{p}:=\left\{\varphi(\Gamma): \varphi \in \mathcal{H}^{\infty}, \Gamma \in \mathcal{G}^{p}\right\}
$$

of $C^{p}$ exact Lagrangian Manifolds isotopic to the zero section. The set

$$
\mathcal{L}:=\left\{\varphi(\Gamma): \varphi \in \mathcal{H}^{\infty}, \Gamma \in \mathcal{G}\right\}
$$

will be especially important for our constructions. Note that

$$
\mathcal{L}=\left\{\varphi(\Gamma): \varphi \in \mathcal{H}^{1}, \Gamma \in \mathcal{G}\right\}
$$

as will be proved in the appendix, hence $\mathcal{L}$ is invariant under $\mathcal{H}^{1}$. Fixing an energy $e \in \mathbb{R}$, we associate to each manifold $L \in \mathcal{L}$ the set

$$
\mathcal{I}_{e}^{*}(L):=\bigcap_{t \in \mathbb{R}} \varphi_{H}^{t}(L \cap\{H=e\}) .
$$

In other words, $\mathcal{I}_{e}^{*}(L)$ is the maximal invariant subset of $L \cap\{H=e\}$. It is compact and invariant, but may in general be empty. Our main result in the present paper is :

Theorem 1. If $H$ is optical, then for each $L \in \mathcal{L}$ contained in $\{H \leqslant e\}$, there exists $\Gamma \in \mathcal{G}$ contained in $\{H \leqslant e\}$ and such that $\mathcal{I}_{e}^{*}(\Gamma)=\mathcal{I}_{e}^{*}(L)$.

Although this result holds for each $e \geqslant \max _{(x, p) \in L} H(x, p)$, the value $e=\max _{(x, p) \in L} H(x, p)$ is more interesting than the others. If $e>\max _{(x, p) \in L} H(x, p)$ then the set $\mathcal{I}_{e}^{*}(L)$ is empty; Theorem 1 for such values of $e$ follows from Theorem 1 for $e=\max _{(x, p) \in L} H(x, p)$. Actually, taking $e=\max _{(x, p) \in L} H(x, p)$ is not sufficient to ensure that the set $\mathcal{I}_{e}^{*}(L)$ is non-empty. It will appear clearly in the sequel that $\mathcal{I}_{e}^{*}(L)$ is non-empty if and only if $e=\alpha(H)$ (this number will be defined below).

Let us now expose two remarkable consequences of this theorem. First, it implies the following generalization of a recent result of Marie-Claude Arnaud [3]:

Theorem 2. If $L \in \mathcal{L}$ is invariant under an optical Hamiltonian, then it belongs to $\mathcal{G}$.

In the case where $L$ is smooth, this result, which had been conjectured several decades ago, has recently been proved by Marie-Claude Arnaud. Partial results involving additional assumptions had been obtained earlier by Bialy and Polterovich, see [4, 5]. Our generalization to Lipschitz manifolds is rather natural since many invariant exact Lagrangian graphs of optical Hamiltonian systems are Lipschitz but not $C^{1}$. 
Proof. If $L$ is invariant, then it is contained in an energy level $\{H=e\}$. To prove this claim, we consider the Lipschitz function $H \circ i: M \longrightarrow \mathbb{R}$, where $i(x)=\varphi\left(x, d v_{x}\right)$. Let $x$ be a point of differentiability of $d v$, hence of $i$. The second differential $d^{2} v_{x}$ is then symmetric, which implies that its graph is a Lagrangian subspace of $T_{\left(x, d v_{x}\right)} T^{*} M$. The linear map $d i_{x}$ is thus a Lagrangian linear embedding of $T_{x} M$ into $T_{i(x)}\left(T^{*} M\right)$ whose image is the tangent plane of $L$ at $q=i(x)$. The invariance of $L$ implies that the Hamiltonian vector field $X_{H}(q)$ belongs to this tangent plane. For each $v \in d i_{x}\left(T_{x} M\right)$, we have

$$
0=\omega_{q}\left(X_{H}(q), v\right)=d H_{q}(v)
$$

where $\omega$ is the symplectic form and where the first equality holds because $d i_{x}\left(T_{x} M\right)$ is Lagrangian. We conclude that $d(H \circ i)(x)=d H_{q} \circ d i_{x}=0$ for each point $x$ of differentiability of $i$. The Lipschitz function $H \circ i$, having its differential almost everywhere equal to zero, is thus constant. As a consequence, we have $\mathcal{I}_{e}^{*}(L)=L$. By Theorem 1 , there exists $\Gamma \in \mathcal{G}$ such that

$$
L=\mathcal{I}_{e}^{*}(L)=\mathcal{I}_{e}^{*}(\Gamma) \subset \Gamma,
$$

thus $L=\Gamma$.

Our second application of Theorem 1, concerns the so-called Aubry and Mañé sets first introduced by Mather in the study of optical Hamiltonians, see for example [19, 18, 13, 16, 17, 14, 6, 20, 9]. The following discussion is a sequel to [1] (although it can be read independently from this paper), itself inspired by [20]. To a Hamiltonian $H: T^{*} M \longrightarrow \mathbb{R}$, we associate its critical value

$$
\alpha(H):=\inf _{L \in \mathcal{L}} \max _{(x, p) \in L} H(x, p) .
$$

In the case where $H$ is optical, it follows from Theorem 1 that

$$
\alpha(H)=\inf _{\Gamma \in \mathcal{G}} \max _{(x, p) \in \Gamma} H(x, p),
$$

as was already proved in [1]. This is a classical definition of the critical value of optical Hamiltonians, introduced in [13]. We define the Aubry set $\mathcal{A}^{*}(H)$ and the Mañé set $\mathcal{N}^{*}(H)$ by

$$
\mathcal{A}^{*}(H):=\bigcap_{L \in \mathcal{L}, L \subset\{H \leqslant \alpha(H)\}} \mathcal{I}_{\alpha(H)}^{*}(L) \quad, \quad \mathcal{N}^{*}(H):=\bigcup_{L \in \mathcal{L}, L \subset\{H \leqslant \alpha(H)\}} \mathcal{I}_{\alpha(H)}^{*}(L) .
$$

These definitions are not the usual ones, but it follows immediately from Theorem 1 that

$$
\mathcal{A}^{*}(H)=\bigcap_{\Gamma \in \mathcal{G}, \Gamma \subset\{H \leqslant \alpha(H)\}} \mathcal{I}_{\alpha(H)}^{*}(\Gamma) \quad, \quad \mathcal{N}^{*}(H)=\bigcup_{\Gamma \in \mathcal{G}, \Gamma \subset\{H \leqslant \alpha(H)\}} \mathcal{I}_{\alpha(H)}^{*}(\Gamma)
$$

in the case where $H$ is optical. This proves that the geometric definitions (1) coincide with the standard ones in the case of optical Hamiltonians, as was already proved in [1] concerning the Aubry set.

Remark. In [1], we defined the Aubry set as

$$
\tilde{\mathcal{A}}(H)=\bigcap_{L \in \mathcal{L}, L \subset\{H \leqslant \alpha(H)\}}(L \cap\{H=\alpha(H)\}),
$$

which is slightly different from (1) at first sight. Let us prove that this is the same set. Since $\mathcal{I}_{\alpha(H)}^{*}(\Gamma) \subset L \cap\{H=\alpha(H)\}$, we have $\mathcal{A}^{*}(H) \subset \tilde{\mathcal{A}}(H)$. On the other hand, we prove in [1] that $\tilde{\mathcal{A}}(H)$ is invariant. Since $\tilde{\mathcal{A}}(H) \subset L \cap\{H=\alpha(H)\}$ for each $L \in \mathcal{L}$ contained in $\{H \leqslant \alpha(H)\}$, we conclude that $\tilde{\mathcal{A}}(H) \subset \mathcal{I}_{\alpha(H)}^{*}(L)$ for these $L$, and thus that $\tilde{\mathcal{A}}(H) \subset \mathcal{A}^{*}(H)$. 
The reason why we introduce the new definitions (1) of the Aubry and Mañé sets is that they involve only natural objects from the symplectic viewpoint. They explain in a convincing way the property of symplectic invariance. More precisely, the following proposition follows immediately from (1), as was already noticed in [1] concerning the Aubry set:

Proposition 1. Let $H$ be a Hamiltonian, not necessarily optical. For each Hamiltonian diffeomorphism $\varphi \in \mathcal{H}^{1}$, we have

$$
\alpha(H \circ \varphi)=\alpha(H) \quad, \quad \varphi\left(\mathcal{A}^{*}(H \circ \varphi)\right)=\mathcal{A}^{*}(H) \quad, \quad \varphi\left(\mathcal{N}^{*}(H \circ \varphi)\right)=\mathcal{N}^{*}(H) .
$$

This statement is valid for the definition (1) of the Aubry and Mañé sets. It is worth recalling here that we can derive conclusions on the "standard" Aubry and Mañé sets only when both Hamiltonians $H$ and $H \circ \varphi$ are optical. Note also that we can treat only Hamiltonian diffeomorphisms here, while the conclusions of Proposition 1 (in the optical case) was proved in [8] for all exact symplectic diffeomorphisms (see also [23] for other symplectic aspects of Aubry-Mather theory). However, the proof of [8] involves different methods and definitions. We believe that the geometric flavor of our present approach offsets the loss of generality of the statement. It is an intriguing question whether these geometric methods can be extended to exact symplectic diffeomorphisms, see [1] for some discussions on that issue.

The connections with the existing literature will be more explicit if we introduce the Lagrangian function $L: T M \longrightarrow \mathbb{R}$ defined by

$$
L(x, v)=\sup _{p \in T_{x}^{*} M}(p \cdot v-H(x, p)) .
$$

A function $u: M \longrightarrow \mathbb{R}$ is said dominated by $L+e$ if the inequality

$$
u(x(t))-u(x(s)) \leqslant \int_{s}^{t} e+L(x(\sigma), \dot{x}(\sigma)) d \sigma
$$

holds for each $s \leqslant t$ and for each $C^{1}$ curve $x:[s, t] \longrightarrow M$. As is proved in [14], it is equivalent to require that the function $u$ is Lipschitz and solves the Hamilton-Jacobi inequality

$$
H(x, d u(x)) \leqslant e
$$

almost everywhere (we say that $u$ is a Lipschitz sub-solution of the equation $H(x, d u(x))=e)$. The curve $x: \mathbb{R} \longrightarrow M$ is said calibrated by $(u, L, e)$ if the inequality (3) is an equality for all $s \leqslant t$ (we say simply calibrated by $u$ when $L$ and $e$ are clear by the context). We denote by $\mathcal{I}_{e}(u)$ the union, in $M$, of the images of all curves $x: \mathbb{R} \longrightarrow M$ calibrated by $(u, L, e)$. A calibrated curve is minimizing, hence its lifting

$$
(x(t), p(t))
$$

is a Hamiltonian trajectory, with $p(t)=\partial_{v} L(x(t), \dot{x}(t))$. We denote by $\mathcal{I}_{e}^{*}(u) \subset T^{*} M$ the union of all Hamiltonian trajectories which project on curves calibrated by $(u, L, e)$. When not empty the set $\mathcal{I}_{e}^{*}(u)$ is compact and invariant. If $u$ is dominated by $L+e$ and $x(t): \mathbb{R} \longrightarrow M$ is calibrated by $u$, then the function $u$ is differentiable at the point $x(t)$ for each $t$, and

$$
\left\{\begin{array}{l}
d u(x(t))=\partial_{v} L(x(t), \dot{x}(t))=p(t) \\
H(x(t), d u(x(t)))=e
\end{array}\right.
$$

This is proved in the Theorem 4.3.8 of [14]. If $u$ is dominated and $C^{1,1}$, then the sets $\mathcal{I}_{e}^{*}(u)$ and $\mathcal{I}_{e}^{*}\left(\Gamma_{u}\right)$ are equal, where $\Gamma_{u}=\{(x, d u(x)), x \in M\}$. The inclusion $\mathcal{I}_{e}^{*}(u) \subset \mathcal{I}_{e}^{*}\left(\Gamma_{u}\right)$ follows from (4). Conversely, let $(x(t), p(t))$ be a Hamiltonian orbit contained in $\Gamma_{u}$. The $(u, L, e)$ calibration of $x(t)$ is the integrated form of the equality

$$
d u(x(t)) \cdot \dot{x}(t)=p(t) \cdot \dot{x}(t)=L(x(t), \dot{x}(t))+H(x(t), p(t))=L(x(t), \dot{x}(t))+e .
$$


In the book of Fathi [14], the Aubry and Mañé sets are defined by

$$
\mathcal{A}^{*}(H)=\bigcap_{u} \mathcal{I}_{\alpha(H)}^{*}(u) \quad, \quad \mathcal{N}^{*}(H)=\bigcup_{u} \mathcal{I}_{\alpha(H)}^{*}(u),
$$

where the intersection and the reunion are taken on the set of all functions dominated by $(u, L, \alpha(H))$. That these expressions define the same sets as (2), hence as (1), follows from the following result of [7] (see also [2]):

Theorem 3. Given $e \in \mathbb{R}$, if $u$ is a Lipschitz sub-solution of $H(x, d u(x))=e$, then there exists a $C^{1,1}$ sub-solution $w$ such that $\mathcal{I}_{e}^{*}(w)=\mathcal{I}_{e}^{*}(u)$.

Since this theorem is not explicitly stated in [7], we prove it in Appendix B. The sequel of the paper is devoted to the proof of Theorem 1. We first need to study in Section 2 the concept of generalized graph selector with more details than we did in [1]. We then expose the proof of Theorem 1 in Section 3. Finally, we comment the definition of the set $\mathcal{L}$ in Appendix $A$ and prove Theorem 3 in Appendix B.

\section{Generalized graph selectors}

The cotangent bundle $\pi: T^{*} M \longrightarrow M$ is equipped with its canonical Liouville form $\lambda=p d q$. An embedding $i: M \longrightarrow T^{*} M$ is called exact if the pullback $i^{*} \lambda$ is exact, or in other words if there exists a function $S: M \longrightarrow \mathbb{R}$ such that $i^{*} \lambda=d S$ on $M$. Let us first recall the standard graph selector principle (see [11, 22]). There is a nice presentation in [20], where the idea of using the graph selector for the circle of problems we are considering is introduced.

Theorem 4. Let $i: M \longrightarrow T^{*} M$ be a smooth exact embedding isotopic to the zero section, then the Lagrangian manifold $L=i(M)$ admits a graph selector. It means that there exists a Lipschitz function $u: M \longrightarrow \mathbb{R}$ and a dense open set $U \subset M$ of full measure, such that $u$ is differentiable on $U$ and such that

$$
(x, d u(x)) \in L \quad \text { and } \quad u(x)=S \circ i^{-1}(x, d u(x))
$$

for each $x \in U$.

We will pay special attention to the embeddings of the form

$$
i(x)=\varphi(x, d v(x))
$$

where $\varphi$ is a smooth Hamiltonian diffeomorphism and $v$ is a function on $M$. In the case where $v$ is smooth, the embedding $i$ given by (5) is exact. Moreover, if $G:[0,1] \times T^{*} M \longrightarrow \mathbb{R}$ is a Hamiltonian generating $\varphi$ (which means that $\varphi$ is the time-one Hamiltonian flow of $G$ ), then the function

$$
S(x)=v(x)+\int_{0}^{1} \lambda_{\varphi_{G}^{s}}(x, d v(x)) \cdot X_{G}\left(\varphi_{G}^{s}(x, d v(x))\right)-G\left(s, \varphi_{G}^{s}(x, d v(x))\right) d s
$$

is a Liouville primitive, meaning that $d S=i^{*} \lambda$. This can be checked by a direct, extremely

classical, computation, see $e . g$. [10]. In the expression above, we have denoted by $X_{G}$ the Hamiltonian vector field of $G$ and by $\varphi_{G}^{t}$ the associated Hamiltonian flow. Note that $\varphi_{G}^{1}=\varphi$, by definition. For less regular functions $v$, we have:

Proposition 2. Let $i$ be the Lipschitz mapping given by (5) where $v$ is $C^{1,1}$ and $\varphi$ is the Hamiltonian diffeomorphism generated by the smooth Hamiltonian $G$. Then, the function $S$ given by (6) is a Liouville primitive in the following sense:

For each Lipschitz curve $y(s):[0, t] \longrightarrow L$ contained in $L=i(M)$, we have

$$
S \circ i^{-1}(y(t))-S \circ i^{-1}(y(0))=\int_{0}^{t} \lambda_{y(s)} \cdot \dot{y}(s) d s
$$


Proof. Each Lipschitz curve $y$ in $L$ is of the form $i \circ x$, where $x$ is a Lipschitz curve on $M$. We have to prove the equality

$$
S(x(t))-S(x(0))=\int_{i \circ x} \lambda
$$

for each Lipschitz curve $x(s):[0, t] \longrightarrow M$. When $v$ and $x$ are smooth the result follows because we have $i^{*} \lambda=d S$. In order to prove the result in the general case, we can assume that the image of the curve $\pi \circ i \circ x$ is contained in the domain of a chart of $M$, else we can cut the curve $x$ in small pieces and prove the result for each of these pieces. We thus work in a chart of $M$. Let $v_{k}$ be a sequence of smooth functions converging to $v$ in $C^{1}(M, \mathbb{R})$ and bounded in $W^{2, \infty}$, which means that their differentials are equi-Lipschitz. Let $S_{k}$ be the function associated to $v_{k}$ by the formula (6), the Hamiltonian $G$ being fixed. Note that the functions $S_{k}$ are equi-Lipschitz and converge uniformly to $S$ as $k \longrightarrow \infty$. We also consider a sequence $x_{k}(s):[0, t] \longrightarrow M$ of smooth equi-Lipschitz curves converging uniformly to $x(s)$. We have the equality

$$
S_{k}\left(x_{k}(t)\right)-S_{k}\left(x_{k}(0)\right)=\int_{i_{k} \circ x_{k}} \lambda
$$

where $i_{k}$ is the map associated to the function $v_{k}$ by (5). Since

$$
S_{k}\left(x_{k}(t)\right)-S_{k}\left(x_{k}(0)\right) \longrightarrow S(x(t))-S(x(0)),
$$

the desired result holds if we prove that

$$
\int_{i_{k} \circ x_{k}} \lambda \longrightarrow \int_{i \circ x} \lambda
$$

We denote by $\left(q_{k}(s), p_{k}(s)\right)$ the curve $i_{k} \circ x_{k}(s)$ (recall that we work in a chart), and by $(q(s), p(s))$ the curve $i \circ x(s)$. The curves $\left(q_{k}, p_{k}\right)$ are equi-Lipschitz and converge uniformly to $(q, p)$. We can suppose also by taking a subsequence that $q_{k}$ is converging to $q$ weakly- $\star$ in the Sobolev space $W^{1, \infty}$. As a consequence, we have

$$
\int_{i_{k} \circ x} \lambda=\int_{0}^{t} p_{k}(s) \cdot \dot{q}_{k}(s) d s \longrightarrow \int_{0}^{t} p(s) \cdot \dot{q}(s) d s=\int_{i \circ x} \lambda .
$$

Corollary 1. If the mapping $\pi \circ i: M \longrightarrow M$ is a bi-Lipschitz homeomorphism, then $i(M)=$ $\Gamma_{u}=\{(x, d u(x)): x \in M\} \in \mathcal{G}$ where $u=S \circ(\pi \circ i)^{-1}$.

Note that $u$ is $C^{1,1}$, while $S$ and $\pi \circ i$ may be only Lipschitz.

Proof. Let us work locally and consider local coordinates $(q, p)$ on $T^{*} M$. The manifold $L=i(M)$ is locally the image of the Lipschitz section $P=p \circ i \circ(\pi \circ i)^{-1}$. Given a local Lipschitz curve $q(t)$ in $M$, we consider its pull-back $x(t)=i^{-1}(q(t), P(q(t)))$. We have

$$
u(q(t))-u(q(0))=S(x(t))-S(x(0))=\int_{0}^{t} P(q(s)) \dot{q}(s) d s .
$$

Since this equality holds for each Lipschitz curve $q$, we conclude that $u$ is $C^{1}$ and that $d u=P$.

In [1], we proved that a generalized graph selector $u$ can be associated to the manifold $L=i(M)$ when $v$ is only $C^{1,1}$. Here we need to be a bit more precise on the relation between the generalized graph selector $u$ and the Liouville primitive $S$. As in [1], we denote by $\hat{K}$ the fiberwise convexification of a set $K \subset T^{*} M$. For each $x \in M$, the set $\hat{K}_{x}:=\hat{K} \cap T_{x}^{*} M$ is the convex hull, in $T_{x}^{*} M$, of the set $K_{x}:=K \cap T_{x}^{*} M$. 
Proposition 3. Let $i$ be the embedding given by (5) where $v$ is $C^{1,1}$ and $\varphi$ is the Hamiltonian diffeomorphism generated by the smooth Hamiltonian $G$. Let us set $L=i(M)$. Then, there exists a Lipschitz function $u$ on $M$ such that $(q, d u(q)) \in \hat{L}$ at each point $q$ of differentiability of $u$ and such that the relation

$$
u(q)=S \circ i^{-1}(q, d u(q))
$$

holds at each point $q$ where $u$ is differentiable and where $d u(q)$ is an extremal point of $\hat{L}_{q}$ (thus a point of $L)$.

Even in the case where $v$ is smooth, this result does not follow immediately from Proposition 2 , because the point $q$ does not necessarily belong to $U$. In this case where $v$ is regular, the result is essentially proved in [3]. Before we pass to the proof we present a Lemma which generalizes a nonsmooth analysis result proved by Clarke on page 63 of [12] (see also Proposition 8.4 of [15]).

Lemma 2. Let $u_{k}: M \longrightarrow \mathbb{R}$ be an equi-Lipschitz sequence of functions converging uniformly to a Lipschitz function $u: M \longrightarrow \mathbb{R}$. Let $A \subset M$ be a set of full Lebesgue measure such that each of the $u_{k}$ is differentiable at each point of $A$. For each $q \in M$ we define the set $K_{q} \subset T_{q}^{*} M$ as the set of limit points of the sequences $\left(q_{k}, d u_{k}\left(q_{k}\right)\right), q_{k} \in A, q_{k} \longrightarrow q$. . We also define its convex hull $\widehat{K}_{q}$ in $T_{q}^{*} M$. Note that $K_{q}$, hence $\hat{K}_{q}$, are compact. If $u$ is differentiable at $q$, then $d u(q) \in \widehat{K}_{q}$.

Note: The statement of the Lemma is slightly corrected compared to the published version of the present paper.

Proof. Let us assume by contradiction that $d u(q)$ does not belong to $\hat{K}_{q}$. Then, there exists a vector $\xi \in T_{q} M$ such that $d u(q) \cdot \xi>1$ and $p \cdot \xi<1$ for each $p \in \hat{K}_{q}$. As a consequence, there exists a neighborhood $\Omega$ of $q$ in $M$, that we identify with a convex open subset of $\mathbb{R}^{n}$ via a chart and an integer $N$ such that

$$
d u_{k}(x) \cdot \xi<1
$$

for each $k \geqslant A$ and each $x \in A \cap \Omega$. By Fubini Theorem, there exists a subset $\tilde{A}$ of $A \cap \Omega$ which has full measure in a neighborhood of $q$, and a positive number $S>0$ such that, for each $x \in \tilde{A}$, the inclusion $x+s \xi \in A \cap \Omega$ holds for almost every $s \in[-S, S]$. We conclude that

$$
u_{k}(x+s \xi)<u_{k}(x)+s
$$

for each $k>N, x \in \tilde{A}, s \in[0, S]$. Since $q$ belongs to the closure of $\tilde{A}$, this implies that

$$
u_{k}(q+s \xi) \leqslant u_{k}(q)+s
$$

for each $k>N, s \in[0, S]$. We can pass to the limit $k \longrightarrow \infty$ in this inequality and obtain that

$$
u(q+s \xi) \leqslant u(q)+s
$$

for each $s \in[0, S]$. This is in contradiction with the fact that $d u(q) \cdot \xi>1$. We conclude that $d u(q) \in \hat{K}$.

Proof of Proposition 3. We prove the existence of the function $u$ as in [1]: We consider a sequence $v_{k}$ regularizing $v$, and associate to it the various objects $i_{k}, S_{k}, L_{k}=i_{k}(M), u_{k}$. Note that the functions $S_{k}$ are equi-bounded, and that the relation

$$
u_{k}(x)=S_{k} \circ i_{k}^{-1}\left(x, d u_{k}(x)\right)
$$

holds almost everywhere, which implies that the functions $u_{k}$ are equi-bounded as well. Moreover, the inclusion

$$
\left(x, d u_{k}(x)\right) \in L_{k}
$$


holds for almost every $x$, which implies that the functions $u_{k}$ are equi-Lipschitz. We can thus assume, by Ascoli Theorem, that the functions $u_{k}$ converge uniformly to a limit $u$. It is useful to fix once and for all a subset $A \subset M$ of full measure such that each of the functions $u_{k}$ are differentiable on $A$ and

$$
\left(x, d u_{k}(x)\right) \in L_{k} \quad, \quad u_{k}(x)=S_{k} \circ i_{k}^{-1}\left(x, d u_{k}(x)\right)
$$

for each $k$ and each $x \in A$.

Let us consider a point $q$ where $u$ is differentiable. By Lemma 2, we obtain that $d u(q) \in \hat{K}_{q}$. We claim that

$$
\hat{K}_{q} \subset \hat{L}_{q}
$$

For the moment, let us assume that (7) holds and derive the proposition. What remains to prove is that $u(q)=S \circ i^{-1}(q, d u(q))$ provided $d u(q)$ is an extremal point of $\hat{L}_{q}$. It follows from (7) that $d u(q)$ is then an extremal point of $\hat{K}_{q}$, hence a point of $K_{q}$. In other words, there exists a sequence $q_{k} \in A$ such that $\left(q_{k}, d u_{k}\left(q_{k}\right)\right) \longrightarrow(q, d u(q))$. The equality

$$
u(q)=S \circ i^{-1}(q, d u(q))
$$

that we want to prove thus follows at the limit $k \longrightarrow \infty$ from the equalities

$$
u_{k}\left(q_{k}\right)=S_{k} \circ i_{k}^{-1}\left(q_{k}, d u_{k}\left(q_{k}\right)\right) .
$$

This proves the proposition, assuming (7). We now prove (7), i.e., we prove that $K_{q} \subset L_{q}$, which implies that $\hat{K}_{q} \subset \hat{L}_{q}$. Each point $(q, p)$ of $K_{q}$ is the limit of a sequence $\left(q_{k}, p_{k}\right)$ of points of $L_{k}$. There exist points $x_{k} \in M$ such that $i_{k}\left(x_{k}\right)=\left(q_{k}, p_{k}\right)$, and we can assume that the sequence $x_{k}$ has a limit $x$. Since $i_{k}$ converges uniformly to $i$, we have $(q, p)=\lim i_{k}\left(x_{k}\right)=i(x) \in L$.

\section{Proof of Theorem 1}

We assume, without loss of generality, that $e=0$. Let us consider an element $L$ of $\mathcal{L}$ contained in $\{H \leqslant 0\}$. By definition, there exists a $C^{1,1}$ function $v$ and a smooth Hamiltonian diffeomorphism $\varphi$ such that $L=\varphi\left(\Gamma_{v}\right)$, where $\Gamma_{v}=\{(x, d v(x)): x \in M\}$. As in Section 2, we denote by $i: M \longrightarrow T^{*} M$ the embedding

$$
i(x):=\varphi(x, d v(x))
$$

in such a way that $i(M)=L$. We proved in section 2 the existence of a Lipschitz Liouville primitive $S: M \longrightarrow \mathbb{R}$, and of a generalized graph selector $u: M \longrightarrow \mathbb{R}$ associated to $S$, see Proposition 3. We claim that

$$
\mathcal{I}_{0}^{*}(u)=\mathcal{I}_{0}^{*}(L) .
$$

Assuming the claim, we can finish the proof of Theorem 1. We apply Theorem 3 and get the existence of a $C^{1,1}$ function $w$ such that $H(x, d w(x)) \leqslant 0$ and such that $\mathcal{I}_{0}^{*}(w)=\mathcal{I}_{0}^{*}(u)$. The exact graph $\Gamma=\{(x, d w(x)): x \in M\}$ satisfies all the conclusions of Theorem 1 .

We have to prove (8). Observe that the function $u$ solves almost everywhere the HamiltonJacobi inequality $H(x, d u(x)) \leqslant 0$, hence it is dominated by the Lagrangian $L$. Let us consider an orbit $(q(t), p(t))$ contained in $\mathcal{I}_{0}^{*}(u)$. This implies that $u$ is differentiable at $q(t)$ for each $t \in \mathbb{R}$, that $d u(q(t))=p(t)$, and that $H(q(t), d u(q(t)))=0$, see (4). By Proposition 3, the point $d u(q(t))$ belongs to the convex hull $\hat{L}_{q(t)}$ of $L_{q(t)}:=L \cap T_{q(t)}^{*} M$ and, since $H(q(t), d u(q(t)))=0$, it is an extremal point of this convex set, hence a point of $L_{q(t)}$. We conclude that $(q(t), p(t)) \in L$, so that

$$
\mathcal{I}_{0}^{*}(u) \subset \mathcal{I}_{0}^{*}(L)
$$


In order to prove the other inclusion, we now consider an orbit $y(t)=(q(t), p(t))$ contained in $\mathcal{I}_{0}^{*}(L)$ (if $\mathcal{I}_{0}^{*}(L)$ is empty, then there is nothing to prove). Let us set

$$
f(t):=u(q(t))-u(q(-t))-\int_{-t}^{t} L(q(\sigma), \dot{q}(\sigma)) d \sigma .
$$

We have to prove that the function $f$ is null, which implies that the curve $q$ is calibrated by $(u, L, 0)$. It is easy to check that $f$ is non-positive and non-increasing, because the function $u$ is dominated by $L$. Moreover, using that $H(y(t))=0$ and that $S$ is a Liouville primitive (see Proposition 2), we can compute (using that $L(q, \dot{q})=p \dot{q}-H(q, p)$ ):

$$
\int_{-t}^{t} L(q(\sigma), \dot{q}(\sigma)) d \sigma=\int_{-t}^{t} p(\sigma) \dot{q}(\sigma) d \sigma=S \circ i^{-1}(y(t))-S \circ i^{-1}(y(-t)),
$$

hence

$$
f(t)=u(q(t))-S \circ i^{-1}(y(t))-u(q(-t))+S \circ i^{-1}(y(-t)) .
$$

Let us choose a sequence $t_{k} \longrightarrow \infty$ such that the curves $y\left(t-t_{k}\right)$ and $y\left(t+t_{k}\right)$ converge uniformly on compact sets to limit curves $\alpha(t)=\left(q_{\alpha}(t), p_{\alpha}(t)\right)$ and $\omega(t)=\left(q_{\omega}(t), p_{\omega}(t)\right)$.

Lemma 3. The asymptotic orbits $\alpha(t)$ and $\omega(t)$ belong to $\mathcal{I}_{0}^{*}(u)$ (and even to $\mathcal{A}^{*}(H)$ ).

Incidentally, this Lemma implies that $\alpha(H)=0$ (the value of $e$ ) unless $\mathcal{I}_{0}^{*}(L)$ is empty. Proof. We prove the statement concerning $\omega(t)$, the other being similar. We have to prove that the curve $q_{\omega}(t)$ is calibrated by the dominated function $u$. We can assume by taking a subsequence that $t_{k+1}-t_{k} \longrightarrow \infty$. Since $S$ is a Liouville primitive, we have

$$
\int_{t_{k-1}}^{t_{k+1}} L(q(s), \dot{q}(s)) d s=S \circ i^{-1}\left(y\left(t_{k+1}\right)\right)-S \circ i^{-1}\left(y\left(t_{k-1}\right)\right) \longrightarrow 0
$$

because both sequences $y\left(t_{k-1}\right)$ and $y\left(t_{k+1}\right)$ converge to $\omega(0)$. As a consequence,

$$
u\left(q\left(t_{k+1}\right)\right)-u\left(q\left(t_{k-1}\right)\right)-\int_{t_{k-1}}^{t_{k+1}} L(q(s), \dot{q}(s)) d s \longrightarrow 0 .
$$

For each fixed $t>0$, we have

$$
\begin{gathered}
0 \geqslant u\left(q\left(t_{k}+t\right)\right)-u\left(q\left(t_{k}-t\right)-\int_{t_{k}-t}^{t_{k}+t} L(q(s), \dot{q}(s)) d s\right. \\
\geqslant u\left(q\left(t_{k+1}\right)\right)-u\left(q\left(t_{k-1}\right)\right)-\int_{t_{k-1}}^{t_{k+1}} L(q(s), \dot{q}(s)) d s
\end{gathered}
$$

when $\mathrm{k}$ is large enough, hence

$$
\begin{aligned}
u\left(q_{\omega}(t)\right)-u\left(q_{\omega}(-t)\right)-\int_{-t}^{t} L\left(q_{\omega}(s), \dot{q}_{\omega}(s)\right) d s & = \\
\lim _{k \longrightarrow \infty}\left(u\left(q\left(t_{k}+t\right)\right)-u\left(q\left(t_{k}-t\right)\right)-\int_{t_{k}-t}^{t_{k}+t} L(q(s), \dot{q}(s)) d s\right) & =0 .
\end{aligned}
$$

This implies that the curve $q_{\omega}(t)$ is calibrated by $(u, L, 0)$, and then that its lifting $\left(q_{\omega}(t), p_{\omega}(t)\right)$ belongs to $\mathcal{I}_{0}^{*}(u)$. In this proof, we have only used that $u$ is a dominated function, we have not used its relation to $S$. So we can apply the proof to all dominated functions, and conclude that $\omega(t) \in \mathcal{A}^{*}(H)$.

The lemma implies that $u$ is differentiable at $q_{\alpha}(0)$ and $q_{\omega}(0)$, that $d u\left(q_{\alpha}(0)\right)=p_{\alpha}(0), d u\left(q_{\omega}(0)\right)=$ $p_{\omega}(0)$, and that $H(\alpha(0))=0=H(\omega(0))$. We then conclude from Proposition 3 that

$$
u\left(q_{\alpha}(0)\right)=S \circ i^{-1}(\alpha(0)) \quad, \quad u\left(q_{\omega}(0)\right)=S \circ i^{-1}(\omega(0)) .
$$


We obtain :

$$
\left.f\left(t_{k}\right) \longrightarrow u\left(q_{\omega}(0)\right)-S \circ i^{-1}(\omega(0))-u\left(q_{\alpha}(0)\right)\right)+S \circ i^{-1}(\alpha(0))=0 .
$$

Since $f$ is non-positive and non-increasing, we conclude that $f$ is identically null. As a consequence, the curve $q$ is calibrated by $(u, L, 0)$.

\section{A Lipschitz exact manifolds isotopic to the zero section}

We return in this appendix to the definition of the sets $\mathcal{L}^{p}$ and $\mathcal{L}$. Note that

$$
\mathcal{L}^{p}:=\left\{\varphi(\Gamma): \varphi \in \mathcal{H}^{\infty}, \Gamma \in \mathcal{G}^{p}\right\}
$$

is just the set of $C^{p}$ exact Lagrangian manifolds isotopic to the zero section. It could have been equally defined as

$$
\mathcal{L}^{p}:=\left\{\varphi\left(\Gamma_{0}\right): \varphi \in \mathcal{H}^{p}\right\},
$$

where $\Gamma_{0}$ is the zero section. The first definition has the advantage of allowing a straightforward extension to the Lipschitz regularity

$$
\mathcal{L}:=\left\{\varphi(\Gamma): \varphi \in \mathcal{H}^{\infty}, \Gamma \in \mathcal{G}\right\}
$$

without bothering on defining a notion of Lipschitz Hamiltonian diffeomorphisms. Since $\mathcal{H}^{\infty}$ is a group, the set $\mathcal{L}$ is invariant under $\mathcal{H}^{\infty}$ in the sense that

$$
L \in \mathcal{L}, \varphi \in \mathcal{H}^{\infty} \Longrightarrow \varphi(L) \in \mathcal{L}
$$

Actually, the set $\mathcal{L}$ is even invariant under $\mathcal{H}^{1}$. This follows from :

\section{Proposition 4.}

$$
\mathcal{L}=\left\{\varphi(\Gamma): \varphi \in \mathcal{H}^{1}, \Gamma \in \mathcal{G}\right\} .
$$

Proof. Let us consider a graph $\Gamma \in \mathcal{G}$ and a diffeomorphism $\varphi \in \mathcal{H}^{1}$. We want to prove that $\varphi(\Gamma) \in \mathcal{L}$. Let us approximate the diffeomorphism $\varphi$ by a smooth Hamiltonian diffeomorphism $\psi \in \mathcal{H}^{\infty}$, in the sense that $\psi^{-1} \circ \varphi$ is $C^{1}$-close to the identity. We have $\varphi(\Gamma)=\psi\left(\psi^{-1} \circ \varphi(\Gamma)\right)$. If $\psi^{-1} \circ \varphi$ is sufficiently $C^{1}$-close to the identity, then $\Gamma^{\prime}:=\psi^{-1} \circ \varphi(\Gamma)$ is an element of $\mathcal{G}$, by Corollary 1 and the fact that $\Gamma$ is Lipschitz. We conclude that $\varphi(\Gamma)=\psi\left(\Gamma^{\prime}\right) \in \mathcal{L}$.

\section{B Regularization of sub-solutions}

In this appendix, we deduce Theorem 3 from the content of [7]. We assume that $e=0$. We define the Lax-Oleinik semi-groups by

$$
\begin{aligned}
& T^{t} u(q)=\min _{x(t)=q}\left(u(x(0))+\int_{0}^{t} L(x(s), \dot{x}(s)) d s\right) \\
& \breve{T}^{t} u(q)=\max _{x(0)=q}\left(u(x(t))-\int_{0}^{t} L(x(s), \dot{x}(s)) d s\right),
\end{aligned}
$$

where the extrema are taken on the set of $C^{1}$ curves $x(s):[0, t] \longrightarrow M$ satisfying $x(t)=q$ in the first expression and $x(0)=q$ in the second. For each sub-solution $u$ and each $t>0$, it is proved in [7] that the function $\breve{T}^{s} T^{t} u$ is a $C^{1,1}$ sub-solution provided $s>0$ is small enough. 
Lemma 4. Given a sub-solution $u$, the functions $u, T^{t} u$ and $\breve{T}^{t} u$ coincide on $\mathcal{I}_{0}(u)$ for each $t \geqslant 0$. As a consequence, we have the inclusions $\mathcal{I}_{0}^{*}(u) \subset \mathcal{I}_{0}^{*}\left(T^{t} u\right)$ and $\mathcal{I}_{0}^{*}(u) \subset \mathcal{I}_{0}^{*}\left(\breve{T}^{t} u\right)$.

Proof. Let $(x(s), p(s))$ be an orbit contained in $\mathcal{I}_{0}^{*}(u)$. We have to prove that the curve $x(s)$ is calibrated by $T^{t} u$ and by $\breve{T}^{t} u$. It is a standard and easy fact, proved for example in [14], that $T^{t} u \geqslant u \geqslant \breve{T}^{t} u$ because $u$ is a sub-solution. On the other hand, given $s \in \mathbb{R}$, we have

$$
\begin{array}{r}
u(x(s))=u(x(s-t))+\int_{s-t}^{s} L(x(\sigma), \dot{x}(\sigma)) d \sigma, \\
T^{t} u(x(s)) \leqslant u(x(s-t))+\int_{s-t}^{s} L(x(\sigma), \dot{x}(\sigma)) d \sigma
\end{array}
$$

hence $T^{t} u(x(s)) \leqslant u(x(s))$, and thus $u(x(s))=T^{t} u(x(s))$. Similarly,

$$
\begin{gathered}
u(x(s))=u(x(s+t))-\int_{s}^{s+t} L(x(\sigma), \dot{x}(\sigma)) d \sigma, \\
\breve{T}^{t} u(x(s)) \geqslant u(x(s+t))-\int_{s}^{s+t} L(x(\sigma), \dot{x}(\sigma)) d \sigma
\end{gathered}
$$

hence $\breve{T}^{t} u(x(s)) \geqslant u(x(s))$, and thus $u(x(s))=\breve{T}^{t} u(x(s))$.

This Lemma implies that

$$
\mathcal{I}_{0}^{*}(u) \subset \mathcal{I}_{0}^{*}\left(\breve{T}^{s} T^{t} u\right)
$$

for each $t, s>0$. Let us now consider two sequences $s_{n}<t_{n}$ of positive numbers such that $t_{n} \longrightarrow 0$ and such that $\breve{T}^{s_{n}} T^{t_{n}} u$ is a $C^{1,1}$ sub-solution for each $n \in \mathbb{N}$. Let $a_{n}$ be a sequence of positive numbers such that

$$
w:=\frac{\sum_{n \geqslant 0} a_{n} \breve{T}^{s_{n}} T^{t_{n}} u}{\sum_{n \geqslant 0} a_{n}}
$$

is a $C^{1,1}$ function. This function is a sub-solution, which coincides with $u$ on $\mathcal{I}_{0}(u)$, so that

$$
\mathcal{I}_{0}^{*}(u) \subset \mathcal{I}_{0}^{*}(w)
$$

An orbit is calibrated by $w$ if and only if it is calibrated by each of the sub-solutions $\breve{T}^{s_{n}} T^{t_{n}} u$. Since $\breve{T}^{s_{n}} T^{t_{n}} u \longrightarrow u$ uniformly when $n \longrightarrow \infty$, this implies that the orbit is also calibrated by $u$. We conclude that $\mathcal{I}_{0}^{*}(w)=\mathcal{I}_{0}^{*}(u)$.

\section{References}

[1] P. Bernard and J. Oliveira dos Santos. A geometric definition of the Aubry-Mather set. Journ. Top. Anal. 2(3): 385-393, 2010.

[2] M. C. Arnaud. Pseudographs and the Lax-Oleinik semi-group: a geometric and dynamical interpretation, Nonlinearity 24(1): 71-79, 2011.

[3] M. C. Arnaud. On a Theorem due to Birkhoff, Geometric and Functional Analysis 20(6): 1307-1316 2010.

[4] M. Bialy and L. Polterovich, Hamiltonian diffeomorphisms and Lagrangian distributions Geometric and Functional Analysis 2(2): 173-210, 1992.

[5] M. Bialy and L. Polterovich, Hamiltonian systems, Lagrangian tori and Birkhoff's theorem, Mathematische Annalen, 292(1): 619-627, 1992.

[6] P. Bernard. Connecting orbits of time dependent lagrangian systems. Ann. Institut Fourier, 52(5): 1533-1568, 2002. 
[7] P. Bernard. Existence of $C^{1,1}$ critical sub-solutions of the Hamilton-Jacobi equation on compact manifolds. Annales scientifiques de l'Ecole normale supérieure, 40(3): 445-452, 2007.

[8] P. Bernard. Symplectic aspects of Mather theory. Duke Mathematical Journal, 136(3): 401-420, 2007.

[9] P. Bernard. The dynamics of pseudographs in convex Hamiltonian systems. J. A. M. S., 21(3): 615-669, 2008.

[10] P. Bernard. The Lax-Oleinik semigroup: a Hamiltonian viewpoint. Lecture notes.

[11] M. Chaperon. Lois de conservation et géométrie symplectique. Comptes rendus de l'Académie des sciences. Série 1, Mathématique, 312(4): 345-348, 1991.

[12] F.H. Clarke. Optimization and nonsmooth analysis. Society for Industrial Mathematics, 1990.

[13] G. Contreras, R. Iturriaga, G.P. Paternain, and M. Paternain. Lagrangian graphs, minimizing measures and Mañé's critical values. Geometric and Functional Analysis, 8(5): 788-809, 1998.

[14] A. Fathi. Weak KAM theorem in Lagrangian dynamics. ghost book.

[15] A. Fathi and E. Maderna. Weak KAM theorem on non compact manifolds. NoDEA : Nonlinear Differential Equations and Applications, 14(1) :1-27, 2007

[16] A. Fathi and A. Siconolfi. Existence of $C^{1}$ critical subsolutions of the Hamilton-Jacobi equation. Inventiones mathematicae, 155(2): 363-388, 2004.

[17] A. Fathi and A. Siconolfi. PDE aspects of Aubry-Mather theory for quasiconvex Hamiltonians. Calculus of Variations and Partial Differential Equations, 22(2): 185-228, 2005.

[18] R. Mané. Lagrangian flows: the dynamics of globally minimizing orbits. Bulletin of the Brazilian Mathematical Society, 28(2): 141-153, 1997.

[19] J.N. Mather. Variational construction of connecting orbits. Annales de l'institut Fourier, 43(5): 1349-1386, 1993.

[20] G.P. Paternain, L.V. Polterovich, and K.F. Siburg. Boundary rigidity for Lagrangian submanifolds, non-removable intersections, and Aubry-Mather theory. Moscow Mathematical Journal, 3(2): 593$619,2003$.

[21] R. T. Rockafellar. Convex analysis, second edition. Princeton University Press, 1972.

[22] C. Viterbo. Symplectic topology as the geometry of generating functions. Mathematische Annalen, 292(1): 685-710, 1992.

[23] C. Viterbo. Symplectic homogenization. Arxiv preprint arXiv:0801.0206, 2007. 